\title{
Sit to stand transfer: performance in rising power, transfer time and sway by age and sex in senior athletes
}

\author{
J B Feland, R Hager, R M Merrill
}

Br J Sports Med 2005;39:e39 (http://www.bjsportmed.com/cgi/content/full/39/11/e39). doi: 10.1136/bjsm.2005.018564

\begin{abstract}
Objective: To observe the differences in performance variables of the sit to stand transfer (as measured on the NeuroCom Balance Master) in a population of senior athletes.

Method: A convenience sample of 173 subjects aged 50 years and older. Data were obtained from voluntary participation in a health fair offered at the annual Huntsman World Senior Games in St George, Utah, USA. All sit to stand tests were performed on the NeuroCom Balance Master. The measured parameters were weight transfer time (WTT), rising power (force exerted to rise), and centre of gravity sway (COG sway) during the rising phase.

Results: A significant difference was found between stratified age groups (50-64 and 65+ years) on rising power. There was also a sex difference in rising power. No significant differences were found in weight transfer time or COG sway. Conclusion: While rising power decreases with increasing age in senior athletes, WTT and COG sway remain similar regardless of age or sex. The maintenance of these other two variables (WTT and COG sway) may be attributable to physical activity and/or participation in sport.
\end{abstract}

$\mathrm{t}$ is widely accepted that ageing and declines in numerous physical performance measures are linked. Previous literature has identified the importance of higher levels of physical activity, and more specifically leg strength, to improved functional ability with ageing. ${ }^{1}$ The ability of an older person to live independently is conditional upon performance of important physical activities of daily living (ADL), such as ambulation and sit to stand transfers. Independent performance of ADL requires a combination of both strength and balance.

Balance and postural control is strongly dependent upon the combined input of the visual, vestibular, and peripheral sensory systems. ${ }^{2}$ Furthermore, declines in strength, range of motion, and reaction time are also hypothesised to affect balance control. ${ }^{3}$ Such declines in balance and functional ability may be a result of decreasing strength and activity. ${ }^{4}$ However, numerous studies have shown that strength training and exercise interventions can improve strength, muscle mass, balance, flexibility, and aerobic capacity in the older adult, and recent reviews have highlighted such findings. ${ }^{56}$

It has been suggested that the master athlete may be an ideal model for studying successful ageing and establishing biomarkers for ageing. ${ }^{7}$ Many studies have reported the relationship between aerobic capacity and ageing, ${ }^{8}{ }^{9}$ but the effect of chronic exercise on muscle mass and function is not as abundant. ${ }^{10}$ Likewise, the performance of master athletes or chronic exercisers on basic balance and functional ability tests has not been reported.
Multiple balance tests (such as timed up and go test, tandem walk, unilateral stance, and sit to stand test) can indicate if there is an increased risk of falls ${ }^{11}$ and decreased physical independence. The sit to stand test (STS) is commonly used in the clinical setting to determine a person's level of transfer function, and can be an indicator of lower extremity strength. ${ }^{12}{ }^{13}$ There is a variety of ways to perform the STS test. ${ }^{14}$ The majority of published STS research has been carried out on deconditioned older people (community dwelling and/or institutionalised).

The purpose of this pilot study was threefold: firstly, to observe how senior athletes performed the STS test as measured by three variables (rising power, weight transfer time (WTT), and centre of gravity (COG) sway); secondly, to compare the results by age classification (50-64 years and $65+$ years) and sex; and thirdly, to establish a "picture" of STS performance by senior athletes to use in the future as a comparison to the performance of community dwelling, less active, and sedentary elderly people, with the eventual goal of being able to show a relationship between activity level and STS performance, and if possible the effect on STS performance variables of chronic participation in different sports.

\section{METHODS}

All subjects gave informed consent and this study was approved by the institutional review board to use human subjects.

Subjects were recruited from the Huntsman World Senior Games, as part of a health fair held in conjunction with the games. In total, 173 senior participants ( 107 men, 66 women) were tested. To qualify subjects had to be participants in one or more sporting activities offered at the World Senior Games, have no history of diabetes or peripheral neuropathy, no history of stroke or other neurological disorder, and no recent history of joint replacement within the past year.

To evaluate the STS movement, all tests were performed on the NeuroCom Balance Master. All STS movements were performed from a seated surface, $406 \mathrm{~mm}$ in height, on a large $457 \mathrm{~mm} \times 1524 \mathrm{~mm}$ force platform. Subjects were allowed one practice trial for familiarisation and for adjusting foot placement. For all STS trials, subjects were instructed to stand up as quickly as possible, but they were not allowed to use arms or hands to push off their legs or the seat surface. Subjects were also instructed to stand as still as possible for 5 seconds following the STS movement as part of the COG sway measurement. The STS procedure was repeated three times.

\section{RESULTS}

Bivariate analyses for discrete and continuous data were performed using SAS software (version 8). Data from the sit

Abbreviations: $A D L$, activities of daily living; $C O G$, centre of gravity; STS, sit to stand; WTT, weight transfer time 
to stand test included time to transfer weight over centre of base of support, rising power, and amount of COG sway. Subjects were categorised into male and female groups and into two different age groups (50-64 years and 65+ years).

A significant difference was found for age and sex with regard to rising power. Men aged 50-64 years generated significantly greater rising power than all other groups. Men $65+$ years generated greater rising power than women $65+$ years $(p=0.018)$, but not women aged 50-64 years $(p=0.5501)$. There was no significant difference in rising power between female age groups $(p=0.1235)$. No significant differences existed between groups for WTT or COG sway.

\section{DISCUSSION}

The declining rising power with increasing age was an expected result from this screening. We believe that this also coincides with the decline in athletic performance seen in ageing athletes. ${ }^{75}$ Even though the rate of decline in strength and other physiological measures are reported as being less in senior athletes than non-athletes, ${ }^{15}$ we do not know at this time if the differences in rising power as measured on the NeuroCom Balance Master that we saw between age and sex groups are less than those that would be found in a population of less active community dwelling elderly people.

Of unique interest is the finding that the two other balance variables in this study (WTT and COG sway) were so similar when prior research has shown significant changes in postural sway and balance with age in a population of community dwelling older people. ${ }^{16}$ Therefore, we expected to see differences in these variables even though prior research has indicated that higher activity levels in older people are associated with better balance and muscle force production. ${ }^{17}$ Frequent exercise has also shown to improve sensorimotor function ${ }^{18}$ and dynamic postural stability, ${ }^{19}$ which reduces the risk of falls in older people. Even moderate exercise in long term home programmes have shown to improve balance and reduce falls in older people with a high risk of falls. ${ }^{20}$ Lord et $a l^{18}$ suggest that studies are needed to conclusively demonstrate the role of exercise on balance and falls.

Our results suggest that a high level of activity appears to help retain the speed of weight transfer and COG sway during the STS manoeuvre in senior athletes. Whether or not a particular level of general physical activity or, participation in sport specifically contributes to maintenance of these variables is unclear. Testing of a larger number of subjects and comparison with sedentary elderly subjects is needed to help clarify the impact of activity, and potentially sport, in maintenance of these STS transfer variables.

\section{Authors' affiliations}

J B Feland, R Hager, R M Merrill, Brigham Young University, Provo, UT, USA

Competing interests: none declared

Correspondence to: Dr J B Feland, Brigham Young University, Provo, UT 84602, USA; brent_feland@byu.edu

Received 4 February 2005

In revised form 4 February 2005

Accepted 6 March 2005

\section{REFERENCES}

1 DiPietro L. The epidemiology of physical activity and physical function in older people. Med Sci Sports Exerc 1996;28:596-600.

2 Lord SR, Clark RD, Webster IW. Postural stability and associated physiological factors in a population of aged persons. J Gerontol 1991;46:M69-76.

3 McChesney JW. Balance testing benefits older adult athletes. Biomechanics 2002;9:29-35

4 Jette AM, Branch LG. The Framingham Disability Study: II. Physical disability among the aging. Am J Public Health 1981;71:1211-16.

5 Keysor JJ, Jette AM. Have we oversold the benefit of late-life exercise? J Gerontol A Biol Sci Med Sci 2001;56:M412-23.

6 Singh MA. Exercise comes of age: rationale and recommendations for a geriatric exercise prescription. J Gerontol A Biol Sci Med Sci 2002;57:M262-82.

7 Bortz WMt, Bortz WM 2nd. How fast do we age? Exercise performance over time as a biomarker. J Gerontol A Biol Sci Med Sci 1996;51:M223-5.

8 Hawkins S, Wiswell R. Rate and mechanism of maximal oxygen consumption decline with aging: implications for exercise training. Sports Med 2003:33:877-88.

9 Rogers MA, Hagberg JM, Martin WH 3rd, et al. ecline in VO2max with aging in master athletes and sedentary men. J Appl Physiol 1990;68:2195-9.

10 Hawkins SA, Wiswell RA, Marcell TJ. Exercise and the master athlete-a model of successful aging? J Gerontol A Biol Sci Med Sci 2003;58:1009-11

11 Morley JE. A fall is a major event in the life of an older person. J Gerontol A Biol Sci Med Sci 2002;57:M492-5.

12 Bohannon RW. Sit-to-stand test for measuring performance of lower extremity muscles. Percept Mot Skills 1995;80:163-6.

13 Salem GJ, Wang MY, Young JT, et al. Knee strength and lower- and higherintensity functional performance in older adults. Med Sci Sports Exerc 2000;32:1679-84

14 Janssen WG, Bussmann HB, Stam HJ. Determinants of the sit-to-stand movement: a review. Phys Ther 2002;82:866-79.

15 Morley JE. The aging athlete. J Gerontol A Biol Sci Med Sci 2000;55:M627-9.

16 Gill J, Allum JH, Carpenter MG, et al. Trunk sway measures of postural stability during clinical balance tests: effects of age. J Gerontol A Biol Sci Med Sci 2001 :56:M438-47.

17 Iverson BD, Gossman MR, Shaddeau SA, et al. Balance performance, force production, and activity levels in noninstitutionalized men 60 to 90 years of age. Phys Ther 1990;70:348-55

18 Lord SR, Ward JA, Williams $\mathrm{P}$, et al. The effect of a 12-month exercise trial on balance, strength, and falls in older women: a randomized controlled trial. J Am Geriatr Soc 1995;43:1198-206.

19 Lord SR, Ward JA, Williams P. Exercise effect on dynamic stability in older women: a randomized controlled trial. Arch Phys Med Rehabil 1996:77:232-6.

20 Barnett A, Smith B, Lord SR, et al. Community-based group exercise improves balance and reduces falls in at-risk older people: a randomised controlled trial. Age Ageing 2003;32:407-14. 